\title{
Global Existence of Cylinder Symmetric Solutions for the Nonlinear Compressible Navier-Stokes Equations
}

\author{
Lan Huang, ${ }^{1}$ Fengxiao Zhai, ${ }^{2}$ and Beibei Zhang ${ }^{3}$ \\ ${ }^{1}$ College of Mathematics and Information Science, North China University of Water Resources and \\ Electric Power, Zhengzhou 450011, China \\ ${ }^{2}$ Department of Physics, Zhengzhou University of Light Industry, Zhengzhou 450002, China \\ ${ }^{3}$ School of Statistics, Capital University of Economics and Business, Beijing 100070, China
}

Correspondence should be addressed to Lan Huang, huanglan82@hotmail.com

Received 4 September 2011; Accepted 6 October 2011

Academic Editor: Meng Fan

Copyright (C) 2011 Lan Huang et al. This is an open access article distributed under the Creative Commons Attribution License, which permits unrestricted use, distribution, and reproduction in any medium, provided the original work is properly cited.

\begin{abstract}
We prove the global existence of cylinder symmetric solutions to the compressible Navier-Stokes equations with external forces and heat source in $R^{3}$ for any large initial data. Some new ideas and more delicate estimates are used to prove this result.
\end{abstract}

\section{Introduction}

In this paper, we study the global existence of cylinder symmetric solutions to the nonlinear compressible Navier-Stokes equations with external forces and heat source in a bounded domain $G=\left\{r \in R^{+}, 0<a<r<b<+\infty\right\}$ of $R^{3}$, where $r$ is the radial variable. In the Eulerian coordinates, the system under consideration are expressed as

$$
\begin{gathered}
\rho_{t}+(\rho u)_{r}+\frac{\rho u}{r}=0, \\
\rho\left(u_{t}+u u_{r}-\frac{v^{2}}{r}\right)+P_{r}-v\left(u_{r}+\frac{u}{r}\right)_{r}=f_{1}(r, t), \\
\rho\left(v_{t}+u v_{r}+\frac{u v}{r}\right)-\mu\left(v_{r}+\frac{v}{r}\right)_{r}=f_{2}(r, t),
\end{gathered}
$$




$$
\begin{gathered}
\rho\left(w_{t}+u w_{r}\right)-\mu\left(w_{r r}+\frac{w_{r}}{r}\right)=f_{3}(r, t), \\
C_{V} \rho\left(\theta_{t}+u \theta_{r}\right)-\kappa\left(\theta_{r r}+\frac{\theta_{r}}{r}\right)+P\left(u_{r}+\frac{u}{r}\right)-Q=g(r, t),
\end{gathered}
$$

where

$$
P=\gamma \rho \theta, \quad Q=\lambda\left(u_{r}+\frac{u}{r}\right)^{2}+\mu\left[\left(v_{r}-\frac{v}{r}\right)^{2}+w_{r}^{2}+2 u_{r}^{2}+2\left(\frac{u}{r}\right)^{2}\right],
$$

and $\rho$ is the mass density, $\theta$ is the absolute temperature, $u, v, w$ are the radial velocity, angular velocity, and axial velocity, respectively, and $\lambda, \mu, v, \gamma, C_{V}, \kappa, \lambda$ are the constants satisfying $\gamma, C_{V}, \kappa, \mu>0,3 \lambda+2 \mu \geq 0(v=\lambda+2 \mu) . f_{1}, f_{2}, f_{3}$, and $g$ represent external forces and heat source, respectively. For system (1.1)-(1.5), we consider the following initial boundary value problem:

$$
\begin{gathered}
\rho(r, 0)=\rho_{0}(r), \quad(u, v, w)(r, 0)=\left(u_{0}, v_{0}, w_{0}\right)(r), \quad \theta(r, 0)=\theta_{0}(r), \quad r \in G, \\
(u, v, w)(a, t)=(u, v, w)(b, t)=0, \quad \theta_{r}(a, t)=\theta_{r}(b, t)=0, \quad t \geq 0 .
\end{gathered}
$$

To show the global existence, it is convenient to transform the system (1.1)-(1.5) to that in the Lagrangian coordinates. The Eulerian coordinates $(r, t)$ are connected to the Lagrangian coordinates $(\xi, t)$ by the relation

$$
r(\xi, t)=r_{0}(\xi)+\int_{0}^{t} \tilde{u}(\xi, \tau) d \tau
$$

where $\tilde{u}(\xi, t)=u(r(\xi, t), t)$ and

$$
r_{0}(\xi)=\eta^{-1}(\xi), \quad \eta(r)=\int_{a}^{r} s \rho_{0}(s) d s, \quad r \in G
$$

It should be noted that if $\inf \left\{\rho_{0}(s): s \in(a, b)\right\}>0$, then $\eta$ is invertible. It follows from (1.1), (1.8), and (1.10) that

$$
\int_{a}^{r} s \rho(s, t) d s=\int_{a}^{r_{0}} s \rho_{0}(s) d s=\xi
$$

and $G$ is transformed into $\Omega=(0, L)$ with

$$
L=\int_{a}^{b} s \rho(s, t) d s=\int_{a}^{b} s \rho_{0}(s), \quad \forall t \geq 0 .
$$


Differentiating (1.11) with respect to $\xi$ yields

$$
\partial_{\xi} r(\xi, t)=r(\xi, t)^{-1} \rho^{-1}(r(\xi, t), t)
$$

In general, for a function $\phi(r, t)=\tilde{\phi}(\xi, t)=\phi(r(\xi, t), t)$, we easily get

$$
\begin{gathered}
\partial_{t} \tilde{\phi}(\xi, t)=\partial_{t} \phi(r, t)+u \partial_{r} \phi(r, t), \\
\partial_{\xi} \tilde{\phi}(\xi, t)=\partial_{r} \phi(r, t) \partial_{\xi} r(\xi, t)=\frac{\partial_{r} \phi(r, t)}{r} \rho^{-1}(r, t) .
\end{gathered}
$$

Without danger of confusion, we denote $(\tilde{\rho}, \tilde{u}, \tilde{v}, \tilde{w}, \tilde{\theta})$ still by $(\rho, u, v, w, \theta)$ and $(\xi, t)$ by $(x, t)$. We set $\tau:=1 / \rho$ to denote the specific volume. Therefore, by virtue of (1.13)-(1.15), system $(1.1)-(1.8)$ in the new variables $(x, t)$ read

$$
\begin{gathered}
\tau_{t}=(r u)_{x} \\
u_{t}=r\left[\frac{v(r u)_{x}-r \theta}{\tau}\right]_{x}+\frac{v^{2}}{r}+f_{1}(r(x, t), t), \\
v_{t}=\mu r\left[\frac{(r v)_{x}}{\tau}\right]_{x}-\frac{u v}{r}+f_{2}(r(x, t), t), \\
w_{t}=\mu r\left[\frac{(r w)_{x}}{\tau}\right]_{x}+\mu \frac{\tau w}{r^{2}}+f_{3}(r(x, t), t), \\
C_{V} \theta_{t}=\kappa\left[\frac{r^{2} \theta_{x}}{\tau}\right]_{x}+\frac{1}{\tau}\left[v(r u)_{x}-\gamma \theta\right](r u)_{x}+\mu \frac{\left[(r v)_{x}\right]^{2}}{\tau}+\mu \frac{r^{2} w_{x}^{2}}{\tau}-2 \mu\left(u^{2}+v^{2}\right)_{x}+g(r(x, t), t),
\end{gathered}
$$

together with

$$
\begin{gathered}
\tau(x, 0)=\tau_{0}(x), \quad(u, v, w)(x, 0)=\left(u_{0}, v_{0}, w_{0}\right)(x), \quad \theta(x, 0)=\theta_{0}(x), \quad x \in[0, L] \\
(u, v, w)(0, t)=(u, v, w)(L, t)=0, \quad \theta_{x}(0, t)=\theta_{x}(L, t)=0, \quad t \geq 0 .
\end{gathered}
$$

By (1.9) and (1.13), we have

$$
\begin{array}{cl}
r(x, t)= & r_{0}(x)+\int_{0}^{t} u(x, s) d s, \quad r_{0}(x)=\left[a^{2}+2 \int_{0}^{x} \tau_{0}(y) d y\right]^{1 / 2}, \\
r_{t}(x, t)=u(x, t), \quad r(x, t) r_{x}(x, t)=\tau(x, t) .
\end{array}
$$

Now let us first recall the related results in the literature. When there were no external forces and heat source, in two or three dimensions, the global existence and large time behavior of smooth solutions to the equations of a viscous polytropic ideal gas have been 
investigated for general domains only in the case of sufficiently small initial data, see, for example, [1-3]. For any large initial data, the global existence of generalized solutions was shown in [4-7]. Recently, Qin [8] proved the exponential stability in $H^{1}$ and $H^{2}$, and Qin and Jiang [9] studied the global existence and exponential stability in $H^{4}$ with smallness of initial total energy.

When there exist external forces and heat forces, for one-dimensional case, the system is isentropic compressible Navier-Stokes equations. Mucha [10] obtained the exponential stability under various boundary conditions, Yanagi [11] established the existence of classical solutions, and Qin and Zhao [12] proved the global existence and asymptotic behavior of solutions for pressure $P=\rho^{\gamma}$ with $\gamma=1$. Later on, Zhang and Fang [13] studied the global existence and uniqueness for $\gamma>1$. For nonisentropic compressible Navier-Stokes equations, Qin and $\mathrm{Yu}$ [14] proved the global existence and asymptotic behavior for perfect gas. In twoor three-dimensional case and the external force and heat source $f \neq 0, g \neq 0$, Qin and Wen [15] proved the global existence of spherically symmetric solutions. In this paper, we will prove the global existence of cylinder symmetric solutions with external forces and heat source in a bounded domain in $R^{3}$.

The notation in this paper will be as follows: $L^{\bar{p}}, 1 \leq \bar{p} \leq+\infty, W^{m, \bar{p}}, m \in N, H^{1}=W^{1,2}$, $H_{0}^{1}=W_{0}^{1,2}$ denote the usual (Sobolev) spaces on $(0, L)$. In addition, $\|\cdot\|_{B}$ denotes the norm in the space $B$; we also put $\|\cdot\|=\|\cdot\|_{L^{2}}$. We denote by $C^{k}(I, B), k \in N_{0}$, the space of $k$ times continuously differentiable functions from $I \subseteq R$ into a Banach space $B$, and likewise by $L^{\bar{p}}(I, B), 1 \leq \bar{p} \leq+\infty$ the corresponding Lebesgue spaces. Subscripts $t$ and $x$ denote the (partial) derivatives with respect to $t$ and $x$, respectively. We use $C_{1}$ to denote the generic positive constant depending on the $H^{1}$-norm of the initial data and time $T$.

We suppose that $f_{i}(r(x, t), t)(i=1,2,3), g(r, t)$ satisfy, for any $T>0$,

$$
\begin{gathered}
f_{i} \in L^{1}\left([0, T], L^{\infty}[0, L]\right) \cap L^{2}\left([0, T], L^{2}[0, L]\right), \\
g(r, t)>0, \quad g \in L^{1}\left([0, T], L^{\infty}[0, L]\right) \cap L^{2}\left([0, T], L^{2}[0, L]\right) .
\end{gathered}
$$

We are now in a position to state our main theorems.

Theorem 1.1. Assume that (1.24)-(1.25) hold; if $\left(\tau_{0}, u_{0}, v_{0}, w_{0}, \theta_{0}\right) \in H^{1}[0, L] \times H_{0}^{1}[0, L] \times$ $H_{0}^{1}[0, L] \times H_{0}^{1}[0, L] \times H^{1}[0, L], \tau_{0}(x)>0, \theta_{0}(x)>0$ on $[0, L]$ and the initial data are compatible with the boundary conditions (1.22), then for problem (1.16)-(1.22) there exists a unique global solution $(\tau, u, v, w, \theta) \in C\left([0, T], H^{1}[0, L] \times H_{0}^{1}[0, L] \times H_{0}^{1}[0, L] \times H_{0}^{1}[0, L] \times H^{1}[0, L]\right)$ such that, for any $T>0$,

$$
\begin{gathered}
0<a \leq r(x, t) \leq b, \quad(x, t) \in[0, L] \times[0, T] \\
0<C_{1}^{-1} \leq \tau(x, t) \leq C_{1}, \quad(x, t) \in[0, L] \times[0, T] \\
\|\tau(t)\|_{H^{1}}^{2}+\|u(t)\|_{H^{1}}^{2}+\|v(t)\|_{H^{1}}^{2}+\|w(t)\|_{H^{1}}^{2}+\|\theta(t)\|_{H^{1}}^{2}+\|r(t)\|_{H^{2}}^{2} \\
+\int_{0}^{t}\left(\|\tau\|_{H^{1}}^{2}+\|u\|_{H^{2}}^{2}+\|v\|_{H^{2}}^{2}+\|w\|_{H^{2}}^{2}+\|\theta\|_{H^{2}}^{2}+\left\|u_{t}\right\|^{2}\right. \\
\left.+\left\|v_{t}\right\|^{2}+\left\|w_{t}\right\|^{2}+\left\|\theta_{t}\right\|^{2}\right)(\tau) d \tau \leq C_{1}, \quad \forall t \in[0, T] .
\end{gathered}
$$




\section{Proof of Theorem 1.1}

In this section we will complete the proof of Theorem 1.1. To this end, we assume that in this section all assumptions in Theorem 1.1 hold. The proof of Theorem can be divided into the following several lemmas.

Lemma 2.1. One has

$$
a=r(0, t) \leq r(x, t) \leq r(L, t)=b, \quad \forall(x, t) \in[0, L] \times[0,+\infty) .
$$

Proof. The proof of (2.1) is borrowed from [6, 8]; please refer to (2.1) in [6] or Lemma 2.1 in [8] for detail.

Lemma 2.2. The global solution $(\tau(t), u(t), v(t), w(t), \theta(t))$ to problems (1.16)-(1.22) satisfies the following estimates:

$$
\begin{gathered}
\int_{0}^{L}\left[\frac{1}{2}\left(u^{2}+v^{2}+w^{2}\right)+C_{V} \theta\right](x, t) d x \leq C_{1} \\
\int_{0}^{L} U(x, t) d x+\int_{0}^{t} \int_{0}^{L}\left(\frac{\kappa r^{2} \theta_{x}^{2}}{\tau \theta^{2}}+\frac{\tau u^{2}}{\theta}+\frac{u_{x}^{2}}{\tau \theta}+\frac{(r u)_{x}^{2}}{\tau \theta}+\frac{w_{x}^{2}}{\tau \theta}+\frac{\left(\tau r^{-1} v-r v_{x}\right)^{2}}{\tau \theta}+\frac{g}{\theta}\right)(x, s) d x d s \leq C_{1},
\end{gathered}
$$

where

$$
U(x, t)=\frac{1}{2}\left(u^{2}+v^{2}+w^{2}\right)+\gamma(\tau-\log \tau-1)+C_{V}(\theta-\log \theta-1) .
$$

Proof. Multiplying (1.17)-(1.19) by $u, v$, and $w$, respectively, adding up the results, and using (1.16), we have

$$
\begin{aligned}
\frac{d}{d t}[ & \left.\frac{1}{2}\left(u^{2}+v^{2}+w^{2}\right)+C_{V} \theta\right] \\
= & {\left[\frac{\kappa r^{2} \theta_{x}}{\tau}+\frac{r u\left(v(r u)_{x}-\gamma \theta\right)}{\tau}+\frac{\mu r v(r v)_{x}}{\tau}+\frac{\mu r^{2} w w_{x}}{\tau}-2 \mu\left(u^{2}+v^{2}\right)\right]_{x} } \\
& +f_{1} u+f_{2} v+f_{3} w+g .
\end{aligned}
$$


Integrating (2.5) with respect to $x$ and $t$ over $Q_{T}=[0, L] \times[0, t](t \in[0, T], \forall T>0)$, using boundary condition (1.22), we obtain

$$
\begin{aligned}
\int_{0}^{L}\left[\frac{1}{2}\left(u^{2}+v^{2}+w^{2}\right)+C_{V} \theta\right] d x= & \int_{0}^{L}\left[\frac{1}{2}\left(u_{0}^{2}+v_{0}^{2}+w_{0}^{2}\right)+C_{V} \theta_{0}\right] d x \\
& +\int_{0}^{t} \int_{0}^{L}\left(f_{1} u+f_{2} v+f_{3} w+g\right)(x, s) d x d s \\
\leq & C_{1}+C_{1} \int_{0}^{t} \int_{0}^{L}\left(u^{2}+v^{2}+w^{2}\right)(x, s) d x d s \\
& +C_{1} \int_{0}^{t} \int_{0}^{L}\left(f_{1}^{2}+f_{2}^{2}+f_{3}^{2}\right)(x, s) d x d s+C_{1} \int_{0}^{t}\|g\|_{L^{\infty}} d s
\end{aligned}
$$

which, by using Gronwall's inequality and (1.24)-(1.25), gives (2.2).

By (1.16)-(1.20), we can easily obtain

$$
\begin{aligned}
U_{t}+\frac{\kappa r^{2} \theta_{x}^{2}}{\tau \theta^{2}}+\frac{v(r u)_{x}^{2}+\mu(r v)_{x}^{2}+\mu r^{2} w_{x}^{2}}{\tau \theta}-\frac{2 \mu\left(u^{2}+v^{2}\right)_{x}}{\theta}+\frac{g}{\theta} \\
=\left[\frac{\kappa(\theta-1) r^{2} \theta_{x}}{\tau \theta}+\frac{r u\left(v(r u)_{x}-r \theta\right)}{\tau}+\frac{\mu r v(r v)_{x}}{\tau}+\frac{\mu r^{2} w w_{x}}{\tau}-2 \mu\left(u^{2}+v^{2}\right)+\gamma r u\right]_{x} \\
\quad+f_{1} u+f_{2} v+f_{3} w+g .
\end{aligned}
$$

Note that constants $v=\lambda+2 \mu$ and

$$
\begin{aligned}
\frac{v(r u)_{x}^{2}+\mu(r v)_{x}^{2}}{\tau \theta}-\frac{2 \mu\left(u^{2}+v^{2}\right)_{x}}{\theta} & =\frac{2 \mu\left(\tau^{2} r^{-2} u^{2}+r^{2} u_{x}^{2}\right)+\lambda(r u)_{x}^{2}}{\tau \theta}+\frac{\mu\left(\tau r^{-1} v-r v_{x}\right)^{2}}{\tau \theta} \\
& \geq C_{1}^{-1}\left(\frac{\tau u^{2}}{\theta}+\frac{u_{x}^{2}+\lambda(r u)_{x}^{2}}{\tau \theta}+\frac{\mu\left(\tau r^{-1} v-r v_{x}\right)^{2}}{\tau \theta}\right) .
\end{aligned}
$$

Integrating (2.7) with respect to $x$ and $t$ over $Q_{T}$, using (1.22), (1.24)-(1.25), and (2.8), we conclude

$$
\begin{aligned}
& \int_{0}^{L} U(x, t) d x+\int_{0}^{t} \int_{0}^{L}\left(\frac{\kappa r^{2} \theta_{x}^{2}}{\tau \theta^{2}}+\frac{\tau u^{2}}{\theta}+\frac{u_{x}^{2}+(r u)_{x}^{2}+w_{x}^{2}+\left(\tau r^{-1} v-r v_{x}\right)^{2}}{\tau \theta}+\frac{g}{\theta}\right) d x d s \\
& \quad \leq C_{1}+\int_{0}^{t} \int_{0}^{L}\left(f_{1} u+f_{2} v+f_{3} w+g\right)(x, s) d x d s \\
& \quad \leq C_{1}+C_{1} \int_{0}^{t} \int_{0}^{L}\left(u^{2}+v^{2}+w^{2}+f_{1}^{2}+f_{2}^{2}+f_{3}^{2}\right)(x, s) d x d s+C_{1} \int_{0}^{t}\|g\|_{L^{\infty}} d s \\
& \quad \leq C_{1} .
\end{aligned}
$$

The proof is complete. 
Next we adapt and modify an idea of Qin and Wen [15] for one-dimensional case to give a representation for $\tau$.

Let

$$
\begin{gathered}
\sigma(x, t):=\frac{v(r u)_{x}-\gamma \theta}{\tau}+\int_{0}^{x}\left(\frac{v^{2}-u^{2}}{r^{2}}+\frac{f_{1}(r(y, t), t)}{r}\right) d y, \\
h(x, t):=\int_{0}^{x} \frac{u_{0}}{r_{0}} d y+\int_{0}^{t} \sigma(x, s) d x .
\end{gathered}
$$

Then, we infer from (1.16) and (1.17) that

$$
h_{x}=\frac{u}{r}, \quad h_{t}=\sigma
$$

By (1.16) and (2.12), we have

$$
(h \tau)_{t}=(r u h)_{x}-u^{2}+v(r u)_{x}-r \theta+\tau \int_{0}^{x}\left(\frac{v^{2}-u^{2}}{r^{2}}+\frac{f_{1}}{r}\right) d x .
$$

Integrating (2.13) with respect to $x$ and $t$ over $Q_{T}$, we obtain

$$
\begin{aligned}
\int_{0}^{L} h \tau d x= & \int_{0}^{L} h_{0} \tau_{0} d x-\int_{0}^{t} \int_{0}^{L}\left(u^{2}+r \theta\right) d x d s+\int_{0}^{t} \int_{0}^{L} \tau \int_{0}^{x}\left(\frac{v^{2}-u^{2}}{r^{2}}+\frac{f_{1}}{r}\right) d y d x d s \\
= & \int_{0}^{L} h_{0} \tau_{0} d x-\int_{0}^{t} \int_{0}^{L}\left(u^{2}+r \theta\right) d x d s+\int_{0}^{t} \int_{0}^{L}\left(r r_{x}\right) \int_{0}^{x}\left(\frac{v^{2}-u^{2}}{r^{2}}+\frac{f_{1}}{r}\right) d y d x d s \\
= & \int_{0}^{L} h_{0} \tau_{0} d x-\int_{0}^{t} \int_{0}^{L}\left(u^{2}+r \theta\right) d x d s+\frac{b^{2}}{2} \int_{0}^{t} \int_{0}^{L}\left(\frac{v^{2}-u^{2}}{r^{2}}+\frac{f_{1}}{r}\right) d x d s \\
& -\int_{0}^{t} \int_{0}^{L}\left(\frac{v^{2}-u^{2}}{2}+\frac{r f_{1}}{2}\right) d x d s
\end{aligned}
$$

where $h_{0}(x):=h(x, 0)$. It follows from integration of (1.16) over $Q_{T}$ and use of (1.22) that

$$
\int_{0}^{L} \tau(x, t) d x=\int_{0}^{L} \tau_{0}(x) d x=\tau^{*}
$$

If we apply the mean value theorem to (2.14) and use (2.15), we conclude there is an $x_{0}(t) \in$ $[0, L]$ such that

$$
h\left(x_{0}(t), t\right)=\frac{1}{\tau^{*}} \int_{0}^{L} h(x, t) \tau(x, t) d x
$$


Therefore, we derive from (2.11), (2.14), and (2.16) that

$$
\begin{aligned}
\int_{0}^{t} \sigma\left(x_{0}(t), s\right) d s= & h\left(x_{0}(t), t\right)-\int_{0}^{x_{0}(t)} \frac{u_{0}}{r_{0}} d x \\
= & -\frac{1}{\tau^{*}} \int_{0}^{t} \int_{0}^{L}\left(\frac{v^{2}+u^{2}}{2}+r \theta+\frac{r f_{1}}{2}\right) d x d s+\frac{b^{2}}{2 \tau^{*}} \int_{0}^{t} \int_{0}^{L}\left(\frac{v^{2}-u^{2}}{r^{2}}+\frac{f_{1}}{r}\right) d x d s \\
& +\frac{1}{\tau^{*}} \int_{0}^{L} h_{0}(x) \tau_{0}(x) d x-\int_{0}^{x_{0}(t)} \frac{u_{0}}{r_{0}} d x .
\end{aligned}
$$

Using (2.17), we will show the representation of specific volume $\tau$.

Lemma 2.3. One has the following representation:

$$
\tau(x, t)=\frac{D(x, t)}{B(x, t)}\left[1+\frac{\gamma}{v} \int_{0}^{t} \frac{\theta(x, s) B(x, s)}{D(x, s)} d s\right], \quad x \in[0, L]
$$

where

$$
\begin{aligned}
& D(x, t)=\tau_{0}(x) \exp \left\{\frac { 1 } { v } \left[\frac{1}{\tau^{*}} \int_{0}^{L} \tau_{0} h_{0} d x-\int_{0}^{x} \frac{u_{0}}{r_{0}} d y+\int_{x_{0}(t)}^{x} \frac{u}{r} d y\right.\right. \\
& \left.\left.\quad-\int_{0}^{t} \int_{0}^{x} \frac{f_{1}}{r} d y d s-\frac{1}{\tau^{*}} \int_{0}^{t} \int_{0}^{L} \frac{r f_{1}}{2} d x d s+\frac{b^{2}}{2 \tau^{*}} \int_{0}^{t} \int_{0}^{L} \frac{f_{1}}{r} d x d s\right]\right\}, \\
& B(x, t)=\exp \left\{\frac { 1 } { v } \left[\frac{1}{\tau^{*}} \int_{0}^{t} \int_{0}^{L}\left(\frac{u^{2}+v^{2}}{2}+r \theta\right) d x d s-\frac{b^{2}}{2 \tau^{*}} \int_{0}^{t} \int_{0}^{L} \frac{v^{2}-u^{2}}{r^{2}} d x d s\right.\right. \\
& \left.\left.+\int_{0}^{t} \int_{0}^{x} \frac{v^{2}-u^{2}}{r^{2}} d x d s\right]\right\} .
\end{aligned}
$$

Proof. By (1.16) and (1.17), we have

$$
\left(\frac{u}{r}\right)_{t}=\sigma_{x}=v(\log \tau)_{t x}-r\left(\frac{\theta}{\tau}\right)_{x}+\frac{v^{2}-u^{2}+r f_{1}}{r^{2}}
$$


Integrating (2.20) over $\left[x_{0}(t), x\right] \times[0, t]$ and using (2.17), we derive

$$
\begin{aligned}
v \log \tau(x, t)-\gamma \int_{0}^{t} \frac{\theta(x, s)}{\tau(x, s)} d s \\
=v \log \tau_{0}(x)+\int_{0}^{t} \sigma\left(x_{0}(t), s\right) d s-\int_{0}^{t} \int_{0}^{x} \frac{v^{2}-u^{2}+r f_{1}}{r^{2}}+\int_{x_{0}(t)}^{x}\left(\frac{u}{r}-\frac{u_{0}}{r_{0}}\right) d y \\
=v \log \tau_{0}(x)-\frac{1}{\tau^{*}} \int_{0}^{t} \int_{0}^{L}\left(\frac{v^{2}+u^{2}}{2}+r \theta+\frac{r f_{1}}{2}\right) d x d s+\frac{b^{2}}{2 \tau^{*}} \int_{0}^{t} \int_{0}^{L}\left(\frac{v^{2}-u^{2}}{r^{2}}+\frac{f_{1}}{r}\right) d x d s \\
\quad+\frac{1}{\tau^{*}} \int_{0}^{L} h_{0}(x) \tau_{0}(x) d x-\int_{0}^{x} \frac{u_{0}}{r_{0}} d x-\int_{0}^{t} \int_{0}^{x} \frac{v^{2}-u^{2}+r f_{1}}{r^{2}} d y d s+\int_{x_{0}(t)}^{x} \frac{u}{r} d y
\end{aligned}
$$

which, when the exponentials are taken, turns into

$$
\frac{B(x, t)}{D(x, t)}=\frac{1}{\tau(x, t)} \exp \left(\frac{\gamma}{v} \int_{0}^{t} \frac{\theta(x, s)}{\tau(x, s)} d s\right)
$$

Multiplying (2.22) by $\gamma \theta / v$ and integrating the resulting equation with respect to $t$, we arrive at

$$
\exp \left(\frac{\gamma}{v} \int_{0}^{t} \frac{\theta(x, s)}{\tau(x, s)} d s\right)=1+\frac{\gamma}{v} \int_{0}^{t} \frac{\theta(x, s) B(x, s)}{D(x, s)} d s
$$

Substituting this into (2.22), we obtain (2.18). The proof is complete.

Lemma 2.4. There are positive constants $\bar{\tau}$ and $\underline{\tau}$, such that, for any $T>0$,

$$
\underline{\tau} \leq \tau(x, t) \leq \bar{\tau}, \quad(x, t) \in[0, L] \times[0, T] .
$$

Proof. Recalling the definition $D(x, t)$, we have by (1.24), Cauchy-Schwarz's inequality, and Lemma 2.1 that

$$
\left|\frac{1}{\tau^{*}} \int_{0}^{t} \int_{0}^{L} \frac{r f_{1}}{2} d x d s-\frac{b^{2}}{2 \tau^{*}} \int_{0}^{t} \int_{0}^{L} \frac{f_{1}}{r} d x d s-\int_{0}^{t} \int_{0}^{x} \frac{f_{1}}{r} d y d s\right| \leq C_{1} \int_{0}^{t}\left\|f_{1}\right\|_{L^{\infty}} \leq C_{1}
$$

which, along with Lemma 2.2, gives

$$
0<C_{1}^{-1} \leq D(x, t) \leq C_{1}, \quad(x, t) \in[0, L] \times[0, T] .
$$

By Lemmas 2.1 and 2.2, we easily obtain, for any $0 \leq s \leq t$,

$$
B(x, t) \leq C_{1}, \quad \frac{B(x, s)}{B(x, t)} \leq \exp \left\{-C_{1}(t-s)\right\} .
$$


Therefore, we derive from (2.2), (2.18), and (2.26)-(2.27) that

$$
\begin{gathered}
\tau(x, t) \geq \frac{D(x, t)}{B(x, t)} \geq \underline{\tau} \\
\tau(x, t) \leq C_{1}\left[1+\int_{0}^{t} \theta e^{-C_{1}(t-s)} d s\right] \\
\leq C_{1}+\int_{0}^{t}\left[\left(\int_{0}^{L} \theta d x\right)^{1 / 2}+\left(\int_{0}^{L} \frac{r^{2} \theta_{x}^{2}}{\tau \theta^{2}} d x\right)^{1 / 2}\left(\int_{0}^{L} \frac{\tau \theta}{r^{2}} d x\right)^{1 / 2}\right]^{2} e^{-C_{1}(t-s)} d s \\
\leq C_{1}+C_{1}\left(1+\int_{0}^{t} \max _{[0, L]} \tau(\cdot, s) \int_{0}^{L} \frac{r^{2} \theta_{x}^{2}}{\tau \theta^{2}} d x d s\right) d s
\end{gathered}
$$

which, by using Gronwall's inequality and (2.28), gives (2.24). The proof is complete.

Remark 1. If the initial data or initial energy are small enough, we can obtain the uniform estimate independent of time $t$ about specific volume $\tau$ under assumptions of external forces. Moreover, we can prove the large-time behavior of solutions.

Lemma 2.5. Under the assumptions of Theorem 1.1, one has, for any $T>0$ and for all $t \in[0, T]$,

$$
\int_{0}^{L}\left(\theta^{2}+u^{4}+v^{4}+w^{4}\right) d x+\int_{0}^{t} \int_{0}^{L}\left(\theta_{x}^{2}+u^{2} u_{x}^{2}+v^{2} v_{x}^{2}+w^{2} w_{x}^{2}\right)(x, s) d x d s \leq C_{1} .
$$

Proof. Multiplying (2.5) by $(1 / 2)\left(u^{2}+v^{2}+w^{2}\right)+C_{V} \theta$ and then integrating the result over $Q_{T}$, we have

$$
\begin{aligned}
\frac{1}{2} \int_{0}^{L}( & \left.\frac{1}{2}\left(u^{2}+v^{2}+w^{2}\right)+C_{V} \theta\right)^{2} d x \\
\leq & C_{1}-\frac{C_{V} \mathcal{k}}{2} \int_{0}^{t} \int_{0}^{L} \frac{r^{2} \theta_{x}^{2}}{\tau} d x d s \\
& +C_{1} \int_{0}^{t} \int_{0}^{L}\left\{\frac{r^{2} u^{2} u_{x}^{2}+r^{2} v^{2} v_{x}^{2}+r^{2} w^{2} w_{x}^{2}}{\tau}+u^{4}+v^{4}+\theta^{2}+\theta^{2} u^{2}\right\} d x d s \\
& +\int_{0}^{t} \int_{0}^{L}\left(f_{1} u+f_{2} v+f_{3} w+g\right)\left(\frac{1}{2}\left(u^{2}+v^{2}+w^{2}\right)+C_{V} \theta\right) d x d s,
\end{aligned}
$$


where

$$
\begin{aligned}
& \int_{0}^{t} \int_{0}^{L} g\left(\frac{1}{2}\left(u^{2}+v^{2}+w^{2}\right)+C_{V} \theta\right) d x d s \\
& \quad \leq \int_{0}^{t}\|g\|_{L^{\infty}} \int_{0}^{L}\left(\frac{1}{2}\left(u^{2}+v^{2}+w^{2}\right)+C_{V} \theta\right) d x d s \leq C_{1}, \\
& \int_{0}^{t} \int_{0}^{L}\left(f_{1} u+f_{2} v+f_{3} w\right)\left(\frac{1}{2}\left(u^{2}+v^{2}+w^{2}\right)+C_{V} \theta\right) d x d s \\
& \quad \leq C_{1} \int_{0}^{t}\left(\left\|f_{1}\right\|_{L^{\infty}}+\left\|f_{2}\right\|_{L^{\infty}}+\left\|f_{3}\right\|_{L^{\infty}}\right) \int_{0}^{L}\left(u^{2}+u^{4}+v^{2}+v^{4}+w^{2}+w^{4}\right) d x d s \\
& \quad+\int_{0}^{t} \int_{0}^{L} \theta^{2} d x d s .
\end{aligned}
$$

Multiplying (1.17) by $u^{3}$ and then integrating the result over $Q_{T}$, we get

$$
\begin{aligned}
\frac{1}{4} \int_{0}^{L} u^{4} d x \leq & C_{1}-\frac{v}{\bar{\tau}} \int_{0}^{t} \int_{0}^{1} r^{2} u^{2} u_{x}^{2} d x d s+C_{1} \int_{0}^{t} \int_{0}^{L}\left(u^{4}+\theta^{2} u^{2}\right)(x, s) d x d s \\
& +\int_{0}^{t} \int_{0}^{L}\left(\frac{v^{2}}{r}+f_{1}\right) u^{3} d x d s \\
\leq & C_{1}-\frac{v}{\bar{\tau}} \int_{0}^{t} \int_{0}^{1} r^{2} u^{2} u_{x}^{2} d x d s+C_{1} \int_{0}^{t} \int_{0}^{L}\left(u^{4}+\theta^{2} u^{2}\right)(x, s) d x d s \\
& +C_{1} \int_{0}^{t}\left(\left\|f_{1}\right\|_{L^{\infty}}+\|u\|_{L^{\infty}}^{2}\right) \int_{0}^{L}\left(v^{4}+u^{2}+u^{4}\right) d x d s .
\end{aligned}
$$

Similarly, multiplying (1.18) and (1.19) by $v^{3}$ and $w^{3}$, respectively, and then integrating over $Q_{T}$, we have

$$
\begin{aligned}
\frac{1}{4} \int_{0}^{L} v^{4} d x & \leq C_{1}-\frac{\mu}{\bar{\tau}} \int_{0}^{t} \int_{0}^{1} r^{2} v^{2} v_{x}^{2} d x d s+C_{1} \int_{0}^{t} \int_{0}^{L}\left(v^{4}+u^{2} v^{4}+f_{2}\left(v^{2}+v^{4}\right)\right) d x d s \\
& \leq C_{1}-\frac{\mu}{\bar{\tau}} \int_{0}^{t} \int_{0}^{1} r^{2} v^{2} v_{x}^{2} d x d s+C_{1} \int_{0}^{t}\left(\left\|f_{2}\right\|_{L^{\infty}}+\|u\|_{L^{\infty}}^{2}+1\right) \int_{0}^{L}\left(v^{2}+v^{4}\right) d x d s, \\
\frac{1}{4} \int_{0}^{L} w^{4} d x & \leq C_{1}-\frac{\mu}{\bar{\tau}} \int_{0}^{t} \int_{0}^{1} r^{2} w^{2} w_{x}^{2} d x d s+C_{1} \int_{0}^{t} \int_{0}^{L}\left(w^{4}+f_{3}\left(w^{2}+w^{4}\right)\right) d x d s \\
& \leq C_{1}-\frac{\mu}{\bar{\tau}} \int_{0}^{t} \int_{0}^{1} r^{2} w^{2} w_{x}^{2} d x d s+C_{1} \int_{0}^{t}\left(\left\|f_{3}\right\|_{L^{\infty}}+1\right) \int_{0}^{L}\left(w^{2}+w^{4}\right) d x d s .
\end{aligned}
$$


Multiplying (2.31) and (2.33) by $\mu /\left(2 \bar{\tau} C_{1}\right)$ and $\mu / \nu$, respectively, adding up the resulting inequalities, and using (2.34) to obtain, with the help of (2.32), the following result:

$$
\begin{aligned}
\int_{0}^{L}\left(u^{4}+v^{4}+w^{4}+\theta^{2}\right) d x+\int_{0}^{t} \int_{0}^{L}\left(\theta_{x}^{2}+u^{2} u_{x}^{2}+v^{2} v_{x}^{2}+w^{2} w_{x}^{2}\right)(x, s) d x d s \\
\leq C_{1}+\int_{0}^{t}\left(\left\|f_{1}\right\|_{L^{\infty}}+\left\|f_{2}\right\|_{L^{\infty}}+\left\|f_{3}\right\|_{L^{\infty}}+1\right) \int_{0}^{L}\left(\theta^{2}+u^{4}+v^{4}+w^{4}\right) d x d s \\
\quad+\int_{0}^{t}\|u\|_{L^{\infty}}^{2} \int_{0}^{L}\left(\theta^{2}+u^{4}+v^{4}+w^{4}\right) d x d s .
\end{aligned}
$$

On the other hand, by (2.3) and (2.20),

$$
\int_{0}^{t}\|u\|_{L^{\infty}}^{2} d s \leq \int_{0}^{t}\left(\int_{0}^{L}\left|u_{x}\right|^{2} d x\right)^{2} d s \leq \int_{0}^{t} \int_{0}^{L} \frac{u_{x}^{2}}{\tau \theta} d x \int_{0}^{L} \tau \theta d x d s \leq C_{1} .
$$

In view of (1.24)-(1.25) and (2.36), we apply Gronwall's inequality to (2.35) to obtain (2.30). The proof is complete.

Lemma 2.6. Under the assumptions of Theorem 1.1, one has, for any $T>0$,

$$
\int_{0}^{L} \tau_{x}^{2}(x, t) d x+\int_{0}^{t} \int_{0}^{L} \theta \tau_{x}^{2}(x, s) d x d s \leq C_{1}, \quad \forall t \in[0, T]
$$

Proof. By means of (1.16), we rewrite (1.17) as

$$
\left(\frac{u}{r}-\frac{v \tau_{x}}{\tau}\right)_{t}=\frac{r\left(\theta \tau_{x}-\tau \theta_{x}\right)}{\tau^{2}}+\frac{v^{2}-u^{2}+r f_{1}}{r^{2}}
$$

Multiplying (2.38) by $(u / r)-\left(v \tau_{x} / \tau\right)$ in $L^{2}[0, L]$ and using Lemmas 2.1-2.5, we arrive at

$$
\begin{aligned}
\frac{1}{2} \frac{d}{d t} \| & \frac{u}{r}-\frac{v \tau_{x}}{\tau} \|^{2}+v \gamma \int_{0}^{L} \frac{\theta \tau_{x}^{2}}{\tau^{2} r} d x \\
& =\int_{0}^{L}\left[\frac{\gamma\left(\theta \tau_{x}-\tau \theta_{x}\right) u}{r \tau^{2}}+\frac{r v \tau_{x} \theta_{x}}{r \tau^{2}}+\frac{v^{2}-u^{2}+r f_{1}}{r^{2}}\left(\frac{u}{r}-\frac{v \tau_{x}}{\tau}\right)\right] d x \\
& \leq \frac{1}{2} v \gamma \int_{0}^{L} \frac{\theta \tau_{x}^{2}}{\tau^{2} r} d x+C_{1} \int_{0}^{L}\left(\theta u^{2}+\theta_{x}^{2}+u^{2}+\frac{\theta_{x}^{2}}{\theta}+u^{4}+v^{4}+f_{1}^{2}+\left(\frac{u}{r}-\frac{v \tau_{x}}{\tau}\right)^{2}\right) d x
\end{aligned}
$$


Journal of Applied Mathematics

Integrating it with respect to $t$, using Lemmas 2.1-2.5, (1.24), and (2.36), we get

$$
\begin{aligned}
\| \frac{u}{r}- & \frac{v \tau_{x}}{\tau} \|^{2}+\int_{0}^{t} \int_{0}^{L} \theta \tau_{x}^{2} d x d s \\
& \leq C_{1}+\int_{0}^{t}\left\|\frac{u}{r}-\frac{v \tau_{x}}{\tau}\right\|^{2} d s+C_{1} \int_{0}^{t} \int_{0}^{L}\left(\theta u^{2}+f_{1}^{2}+\theta_{x}^{2}+\frac{\theta_{x}^{2}}{\theta}\right) d x d s \\
& \leq C_{1}+\int_{0}^{t}\left\|\frac{u}{r}-\frac{v \tau_{x}}{\tau}\right\|^{2} d s+\int_{0}^{t}\|u\|_{L^{\infty}}^{2} \int_{0}^{L} \theta d x d s+\int_{0}^{t} \int_{0}^{L} \theta_{x}^{2}\left(1+\frac{1}{\theta^{2}}\right) d x d s \\
& \leq C_{1}+\int_{0}^{t}\left\|\frac{u}{r}-\frac{v \tau_{x}}{\tau}\right\|^{2} d s .
\end{aligned}
$$

We exploit the Gronwall inequality to (2.40) to obtain (2.37). The proof is complete.

Lemma 2.7. Under the assumptions of Theorem 1.1, one has, for any $T>0$,

$$
\begin{aligned}
& \int_{0}^{L} u_{x}^{2}(x, t) d x+\int_{0}^{t} \int_{0}^{L}\left(u_{t}^{2}+u_{x x}^{2}\right)(x, s) d x d s \leq C_{1}, \quad \forall t \in[0, T], \\
& \int_{0}^{L} v_{x}^{2}(x, t) d x+\int_{0}^{t} \int_{0}^{L}\left(v_{t}^{2}+v_{x x}^{2}\right)(x, s) d x d s \leq C_{1}, \quad \forall t \in[0, T], \\
& \int_{0}^{L} w_{x}^{2}(x, t) d x+\int_{0}^{t} \int_{0}^{L}\left(w_{t}^{2}+w_{x x}^{2}\right)(x, s) d x d s \leq C_{1}, \quad \forall t \in[0, T] .
\end{aligned}
$$

Proof. Multiplying (1.17) by $u_{t}$, integrating the result over $Q_{T}$, using Lemmas 2.1-2.6, and taking into account that $(r u)_{x}\left(r u_{t}\right)_{x} / \tau=(1 / 2)\left((r u)_{x}^{2} / \tau\right)_{t}-\left((r u)_{x}\left(u^{2}\right)_{x} / \tau\right)+\left((r u)_{x}^{3} / 2 \tau^{2}\right)$, we obtain

$$
\begin{aligned}
\int_{0}^{t} \int_{0}^{L} u_{t}^{2} d x d s \\
=-\int_{0}^{t} \int_{0}^{L}\left(r u_{t}\right)_{x} \frac{v(r u)_{x}}{\tau}-\int_{0}^{t} \int_{0}^{L}\left(\frac{r \theta}{\tau}\right)_{x} r u_{t} d x d s+\int_{0}^{t} \int_{0}^{L}\left(\frac{v^{2}}{r}+f_{1}\right) u_{t} d x d s \\
=\frac{v}{2} \int_{0}^{L} \frac{\left(r_{0} u_{0}\right)_{x}^{2}}{\tau_{0}} d x-\frac{v}{2} \int_{0}^{L} \frac{(r u)_{x}^{2}}{\tau} d x+r \int_{0}^{t} \int_{0}^{L} r \frac{\tau \theta_{x}-\theta \tau_{x}}{\tau^{2}} u_{t} d x d s \\
\quad-v \int_{0}^{t} \int_{0}^{L} u^{2}\left(\frac{(r u)_{x}}{\tau}\right)_{x} d x d s+\frac{v}{2} \int_{0}^{t} \int_{0}^{L} r u\left(\frac{(r u)_{x}^{2}}{\tau^{2}}\right) d x d s+\int_{0}^{t} \int_{0}^{L}\left(\frac{v^{2}}{r}+f_{1}\right) u_{t} d x d s \\
\leq C_{1}-\frac{v}{2} \int_{0}^{L} \frac{(r u)_{x}^{2}}{\tau} d x+\frac{1}{4} \int_{0}^{t} \int_{0}^{L} u_{t}^{2} d x d s+C_{1} \int_{0}^{t} \int_{0}^{L}\left(\theta^{2} \tau_{x}^{2}+\theta_{x}^{2}+f_{1}^{2}+v^{4}\right) d x d s
\end{aligned}
$$




$$
\begin{aligned}
& -v \int_{0}^{t} \int_{0}^{L} u^{2}\left[\frac{u_{t}}{r}+\frac{1}{r}\left(\frac{r \theta}{\tau}\right)_{x}-\frac{v^{2}+f_{1} r}{r^{2}}\right] d x d s \\
& +\frac{v}{2} \int_{0}^{t} \int_{0}^{L} 2 r u \frac{(r u)_{x}}{\tau}\left[\frac{u_{t}}{r}+\frac{1}{r}\left(\frac{r \theta}{\tau}\right)_{x}-\frac{v^{2}+f_{1} r}{r^{2}}\right] d x d s \\
& \leq C_{1}-\frac{v}{2} \int_{0}^{L} \frac{(r u)_{x}^{2}}{\tau} d x+\frac{1}{2} \int_{0}^{t} \int_{0}^{L} u_{t}^{2} d x d s+C_{1} \int_{0}^{t} \int_{0}^{L}\left(\theta^{2} \tau_{x}^{2}+\theta_{x}^{2}+f_{1}^{2}+v^{4}+u^{4}\right) d x d s \\
& +C_{1} \int_{0}^{t} \int_{0}^{L} u^{2} \frac{(r u)_{x}^{2}}{\tau}(x, s) d x d s .
\end{aligned}
$$

We derive from (2.30) that

$$
\|\theta\|_{L^{\infty}}^{2} \leq \int_{0}^{L} \theta^{2} d x+2 \int_{0}^{L}\left|\theta \theta_{x}\right| d x \leq C_{1} \int_{0}^{L}\left(\theta_{x}^{2}+\theta^{2}\right) d x \leq C_{1}+C_{1} \int_{0}^{L} \theta_{x}^{2} d x .
$$

Therefore, using (1.24), (2.30), (2.36), (2.37), and (2.44)-(2.45), we conclude

$$
\int_{0}^{L} \frac{(r u)_{x}^{2}}{\tau} d x+\int_{0}^{t} \int_{0}^{L} u_{t}^{2} d x d s \leq C_{1}+C_{1} \int_{0}^{t}\|\theta\|_{L^{\infty}}^{2} \int_{0}^{L} \tau_{x}^{2} d x d s+C_{1} \int_{0}^{t}\|u\|_{L^{\infty}}^{2} \int_{0}^{L} \frac{(r u)_{x}^{2}}{\tau}(x, s) d x d s
$$

which, by applying the Gronwall inequality, implies

$$
\int_{0}^{L} u_{x}^{2}(x, t) d x+\int_{0}^{t} \int_{0}^{L} u_{t}^{2}(x, s) d x d s \leq C_{1}
$$

By (1.17), we have

$$
\begin{aligned}
\int_{0}^{t} \int_{0}^{L} u_{x x}^{2}(x, s) d x d s & \leq C_{1} \int_{0}^{t} \int_{0}^{L}\left(u_{t}^{2}+u_{x}^{2}+u^{2}+\tau_{x}^{2} u_{x}^{2}+\theta^{2} \tau_{x}^{2}+\theta_{x}^{2}+v^{4}+f_{1}^{2}\right)(x, s) d x d s \\
& \leq C_{1}+C_{1} \int_{0}^{t}\left(\|\theta\|_{L^{\infty}}^{2}+\left\|u_{x}\right\|_{L^{\infty}}^{2}\right) \int_{0}^{L} \tau_{x}^{2} d x d s \\
& \leq C_{1}+C_{1} \int_{0}^{t}\left(1+\left\|\theta_{x}\right\|^{2}+\left\|u_{x}\right\|\left\|u_{x x}\right\|+\left\|u_{x}\right\|\right)(s) d s \\
& \leq C_{1}+\frac{1}{2} \int_{0}^{t} \int_{0}^{L} u_{x x}^{2}(x, s) d x d s .
\end{aligned}
$$


Therefore,

$$
\int_{0}^{t}\left\|u_{x x}(s)\right\|^{2} d s \leq C_{1}
$$

which, along with (2.47), gives (2.41).

Analogously, multiplying (1.18) by $v_{t}$, integrating the result over $Q_{T}$, and using assumptions (1.24) and Lemmas 2.1-2.6, we deduce

$$
\begin{aligned}
\frac{\mu}{2} \int_{0}^{L} \frac{(r v)_{x}^{2}}{\tau} d x+\int_{0}^{t}\left\|v_{t}(s)\right\|^{2} d s \\
=\frac{\mu}{2} \int_{0}^{L} \frac{\left(r_{0} v_{0}\right)_{x}^{2}}{\tau_{0}} d x+\frac{\mu}{2} \int_{0}^{t} \int_{0}^{L} r u\left[\frac{(r v)_{x}^{2}}{\tau}\right]_{x} d x d s \\
\quad-\mu \int_{0}^{t} \int_{0}^{L}\left[\frac{(r v)_{x}}{\tau}\right]_{x} u v d x d s-\int_{0}^{t} \int_{0}^{L} \frac{u v v_{t}}{r} d x d s+\int_{0}^{t} \int_{0}^{L} f_{2} v_{t} d x d s \\
\leq C_{1}+\frac{1}{4} \int_{0}^{t}\left\|v_{t}(s)\right\|^{2} d s-\frac{1}{2} \int_{0}^{t} \int_{0}^{L} u \frac{(r v)_{x}}{\tau}\left[v_{t}+\frac{u v}{r}-f_{2}\right] d x d s \\
\quad-\int_{0}^{t} \int_{0}^{L} u v\left[\frac{v_{t}-f_{2}}{r}+\frac{u v}{r^{2}}\right] d x d s \\
\leq C_{1}+\frac{1}{2} \int_{0}^{t}\left\|v_{t}(s)\right\|^{2} d s+\int_{0}^{t}\|u\|_{L^{\infty}}^{2} \int_{0}^{L} \frac{(r v)_{x}^{2}}{\tau} d x d s+C_{1} \int_{0}^{t} \int_{0}^{L}\left(u^{2} v^{2}+f_{2}^{2}\right) d x d s \\
\leq C_{1}+\frac{1}{2} \int_{0}^{t}\left\|v_{t}(s)\right\|^{2} d s+\int_{0}^{t}\|u\|_{L^{\infty}}^{2} \int_{0}^{L}\left(v^{2}+\frac{(r v)_{x}^{2}}{\tau}\right) d x d s .
\end{aligned}
$$

In view of (2.36), we apply Gronwall's inequality to (2.50) to obtain

$$
\int_{0}^{L} \frac{(r v)_{x}^{2}}{\tau} d x+\int_{0}^{t} \int_{0}^{L} v_{t}^{2}(x, s) d x d s \leq C_{1}
$$

By (1.18) and (2.51), we easily deduce

$$
\int_{0}^{t} \int_{0}^{L} v_{x x}^{2}(x, s) d x d s \leq C_{1}
$$

which, along with (2.51) and Lemmas 2.1-2.4, implies (2.42). The proof of (2.43) iS similar to that of (2.41) and (2.42). The proof is now complete.

Lemma 2.8. Under the assumptions of Theorem 1.1, one has, for any $T>0$,

$$
\int_{0}^{L} \theta_{x}^{2}(x, t) d x+\int_{0}^{t} \int_{0}^{L}\left(\theta_{t}^{2}+\theta_{x x}^{2}\right)(x, s) d x d s \leq C_{1}, \quad \forall t \in[0, T] .
$$


Proof. Multiplying (1.20) by $\theta_{t}$ over $Q_{T}$, we have

$$
\begin{aligned}
& \frac{\kappa}{2} \int_{0}^{L} \frac{r^{2} \theta_{x}^{2}}{\tau} d x+C_{V} \int_{0}^{t} \int_{0}^{L} \theta_{t}^{2}(x, s) d x d s \\
& =\frac{\kappa}{2} \int_{0}^{L} \frac{r_{0}^{2} \theta_{0 x}^{2}}{\tau_{0}} d x+\frac{\kappa}{2} \int_{0}^{t} \int_{0}^{L}\left(\frac{r^{2}}{\tau}\right) \theta_{x}^{2} d x d s \\
& +\int_{0}^{t} \int_{0}^{L}\left[\frac{1}{\tau}\left[v(r u)_{x}-r \theta\right](r u)_{x}+\mu \frac{\left[(r v)_{x}\right]^{2}}{\tau}+\mu \frac{r^{2} w_{x}^{2}}{\tau}-2 \mu\left(u^{2}+v^{2}\right)_{x}\right. \\
& \quad+g(r(x, t), t)] \theta_{t}(x, s) d x d s .
\end{aligned}
$$

Using Lemmas 2.1-2.7, the Cauchy-Schwarz inequality, and the interpolation inequality, we have

$$
\begin{aligned}
& \left|\frac{\kappa}{2} \int_{0}^{t} \int_{0}^{L}\left(\frac{r^{2}}{\tau}\right) \theta_{x}^{2} d x d s\right| \\
& \leq C_{1} \int_{0}^{t}\left(\|u\|_{L^{\infty}}+\left\|(r u)_{x}\right\|_{L^{\infty}}\right) \int_{0}^{L} \theta_{x}^{2} d x d s \\
& \leq C_{1} \int_{0}^{t}\left(\left\|u_{x}\right\|+\left\|(r u)_{x}\right\|^{1 / 2}\left\|(r u)_{x x}\right\|^{1 / 2}\right) \int_{0}^{L} \theta_{x}^{2} d x d s \\
& \leq C_{1} \int_{0}^{t}\left(\left\|u_{x}\right\|+\left\|(r u)_{x x}\right\|^{2}+\left\|(r u)_{x}\right\|^{2 / 3}\right) \int_{0}^{L} \theta_{x}^{2} d x d s \\
& \leq C_{1} \int_{0}^{t} \int_{0}^{L} \theta_{x}^{2} d x d s+C_{1} \int_{0}^{t}\left(\int_{0}^{L}\left(u^{2} \tau_{x}^{2}+u_{x}^{2}+u^{2}+u_{x x}^{2}\right) d x\right) \int_{0}^{L} \theta_{x}^{2} d x d s \\
& \leq C_{1}+C_{1} \int_{0}^{t}\left(\|u\|_{L^{\infty}}^{2}\left\|\tau_{x}\right\|^{2}+\|u\|^{2}+\left\|u_{x x}\right\|^{2}\right) \int_{0}^{L} \theta_{x}^{2} d x d s \\
& \leq C_{1}+C_{1} \int_{0}^{t}\left(\|u\|_{L^{\infty}}^{2}+\left\|u_{x x}\right\|^{2}\right) \int_{0}^{L} \theta_{x}^{2} d x d s \\
& \left|\int_{0}^{t} \int_{0}^{L}\left[\frac{1}{\tau}\left[v(r u)_{x}-\gamma \theta\right](r u)_{x}+\mu \frac{\left[(r v)_{x}\right]^{2}}{\tau}+\mu \frac{r^{2} w_{x}^{2}}{\tau}-2 \mu\left(u^{2}+v^{2}\right)_{x}+g(r, t)\right] \theta_{t} d x d s\right| \\
& \leq \frac{1}{4} \int_{0}^{t}\left\|\theta_{t}\right\|^{2} d s+C_{1} \int_{0}^{t} \int_{0}^{L}\left[(r u)_{x}^{4}+(r v)_{x}^{4}+\theta^{2}(r u)_{x}^{2}+w_{x}^{4}+u^{2} u_{x}^{2}+v^{2} v_{x}^{2}+g^{2}\right] d x d s
\end{aligned}
$$




$$
\begin{aligned}
& \leq \frac{1}{4} \int_{0}^{t}\left\|\theta_{t}\right\|^{2} d s+C_{1} \int_{0}^{t}[\left\|(r u)_{x}\right\|_{L^{\infty}}^{2}\left(\left\|(r u)_{x}\right\|^{2}+\|\theta\|^{2}\right)+\left\|(r v)_{x}\right\|_{L^{\infty}}^{2}\left\|(r v)_{x}\right\|^{2} \\
&\left.+\left\|w_{x}\right\|_{L^{\infty}}^{2}\left\|w_{x}\right\|^{2}\right](s) d s+C_{1} \int_{0}^{t} \int_{0}^{L}\left(u^{2} u_{x}^{2}+v^{2} v_{x}^{2}+g^{2}\right)(x, s) d x d s .
\end{aligned}
$$

Similarly to (2.55), by virtue of (1.25), (2.30), and (2.41)-(2.43), we arrive at

$$
\begin{aligned}
& \mid \int_{0}^{t} \int_{0}^{L} {\left[\frac{1}{\tau}\left[v(r u)_{x}-r \theta\right](r u)_{x}+\mu \frac{\left[(r v)_{x}\right]^{2}}{\tau}+\mu \frac{r^{2} w_{x}^{2}}{\tau}-2 \mu\left(u^{2}+v^{2}\right)_{x}+g(r, t)\right] \theta_{t} d x d s \mid } \\
& \quad \leq C_{1}+\frac{1}{4} \int_{0}^{t}\left\|\theta_{t}\right\|^{2} d s+C_{1} \int_{0}^{t}\left(\left\|(r u)_{x}\right\|_{L^{\infty}}^{2}+\left\|(r v)_{x}\right\|_{L^{\infty}}^{2}+\left\|w_{x}\right\|_{L^{\infty}}^{2}\right) \\
& \quad \leq C_{1}+\frac{1}{4} \int_{0}^{t}\left\|\theta_{t}\right\|^{2} d s+C_{1} \int_{0}^{t}\left(\left\|\tau_{x}\right\|^{2}+\left\|u_{x}\right\|_{H^{1}}^{2}+\left\|v_{x}\right\|_{H^{1}}^{2}+\left\|w_{x}\right\|_{H^{1}}^{2}\right) \\
& \quad \leq C_{1}+\frac{1}{4} \int_{0}^{t}\left\|\theta_{t}\right\|^{2} d s .
\end{aligned}
$$

Combining (2.54)-(2.57), we conclude

$$
\left\|\theta_{x}\right\|^{2}+\int_{0}^{t}\left\|\theta_{t}(s)\right\|^{2} d s \leq C_{1}+C_{1} \int_{0}^{t}\left(\|u\|_{L^{\infty}}^{2}+\left\|u_{x x}\right\|^{2}\right)\left\|\theta_{x}\right\|^{2} d s
$$

In view of (2.36) and (2.41), we apply Gronwall's inequality to (2.58) to obtain

$$
\left\|\theta_{x}(t)\right\|^{2}+\int_{0}^{t}\left\|\theta_{t}(s)\right\|^{2} d s \leq C_{1}, \quad \forall t \in[0, T]
$$

Similarly to proof of (2.41), by Lemmas 2.1-2.7, (1.20), (1.25), and (2.59), we obtain

$$
\int_{0}^{t}\left\|\theta_{x x}(s)\right\|^{2} d s \leq C_{1}
$$

which, together with (2.59), implies (2.53). The proof is complete.

Proof of Theorem 1.1. By Lemmas 2.1-2.8, we complete the proof of Theorem 1.1.

\section{Acknowledgments}

The work is in part supported by Doctoral Foundation of North China University of Water Sources and Electric Power (no. 201087) and the Natural Science Foundation of Henan Province of China (no. 112300410040). 


\section{References}

[1] G.-Q. Chen, D. Hoff, and K. Trivisa, "Global solutions of the compressible Navier-Stokes equations with large discontinuous initial data," Communications in Partial Differential Equations, vol. 25, no. 1112 , pp. 2233-2257, 2000.

[2] A. Matsumura and T. Nishida, "Initial-boundary value problems for the equations of motion of compressible viscous and heat-conductive fluids," Communications in Mathematical Physics, vol. 89, no. 4, pp. 445-464, 1983.

[3] A. Matsummura and M. Padula, "Stability of stationary flow of compressible fluids to large external potential forces," SAACM, vol. 2, pp. 183-202, 1992.

[4] H. Fujita-Yashima and R. Benabidallah, "Équation à symétrie sphérique d'un gaz visqueux et calorifère avec la surface libre," Annali di Matematica Pura ed Applicata, vol. 168, pp. 75-117, 1995.

[5] S. Jiang, "Global spherically symmetric solutions to the equations of a viscous polytropic ideal gas in an exterior domain," Communications in Mathematical Physics, vol. 178, no. 2, pp. 339-374, 1996.

[6] S. Jiang, "Large-time behavior of solutions to the equations of a viscous polytropic ideal gas," Annali di Matematica Pura ed Applicata, vol. 175, pp. 253-275, 1998.

[7] S. Zheng and Y. Qin, "Universal attractors for the Navier-Stokes equations of compressible and heatconductive fluid in bounded annular domains in $R^{n}$," Archive for Rational Mechanics and Analysis, vol. 160, no. 2, pp. 153-179, 2001.

[8] Y. Qin, "Exponential stability for the compressible Navier-Stokes equations with the cylinder symmetry in $R^{3}$," Nonlinear Analysis: Real World Applications, vol. 11, no. 5, pp. 3590-3607, 2010.

[9] Y. Qin and L. Jiang, "Global existence and exponential stability of solutions in $H^{4}$ for the compressible Navier-Stokes equations with the cylinder symmetry," Journal of Differential Equations, vol. 249, no. 6, pp. 1353-1384, 2010.

[10] P. B. Mucha, "Compressible Navier-Stokes system in 1-D," Mathematical Methods in the Applied Sciences, vol. 24, no. 9, pp. 607-622, 2001.

[11] S. Yanagi, "Existence of periodic solutions for a one-dimensional isentropic model system of compressible viscous gas," Nonlinear Analysis: Theory, Methods \& Applications A, vol. 46, no. 2, pp. 279-298, 2001.

[12] Y. Qin and Y. Zhao, "Global existence and asymptotic behavior of the compressible Navier-Stokes equations for a 1D isothermal viscous gas," Mathematical Models \& Methods in Applied Sciences, vol. 18, no. 8, pp. 1383-1408, 2008.

[13] T. Zhang and D. Fang, "Global behavior of compressible Navier-Stokes equations with a degenerate viscosity coefficient," Archive for Rational Mechanics and Analysis, vol. 182, no. 2, pp. 223-253, 2006.

[14] Y. Qin and X. Yu, "Global existence and asymptotic behavior for the compressible Navier-Stokes equations with a non-autonomous external force and a heat source," Mathematical Methods in the Applied Sciences, vol. 32, no. 8, pp. 1011-1040, 2009.

[15] Y. Qin and S. Wen, "Global existence of spherically symmetric solutions for nonlinear compressible Navier-Stokes equations," Journal of Mathematical Physics, vol. 49, no. 2, Article ID 023101, 2008. 


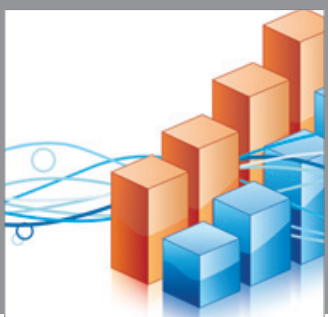

Advances in

Operations Research

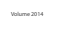

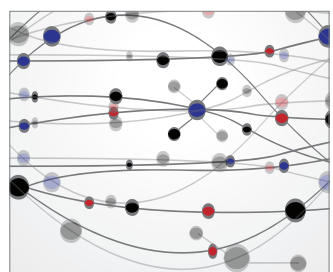

\section{The Scientific} World Journal
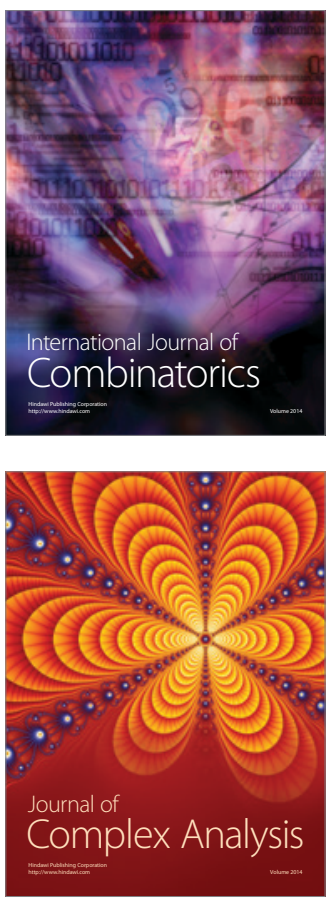

International Journal of

Mathematics and

Mathematical

Sciences
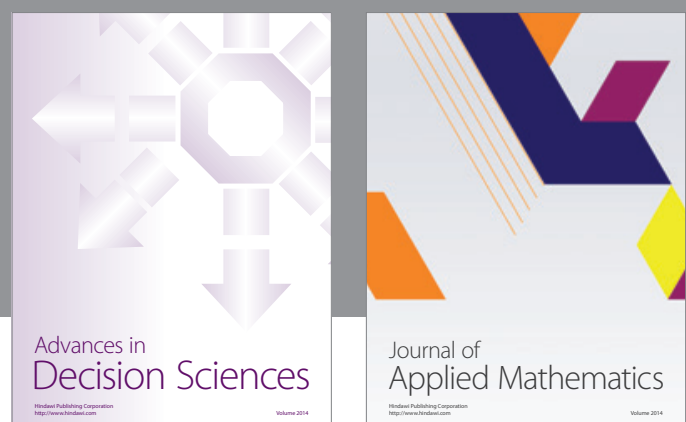

Journal of

Applied Mathematics
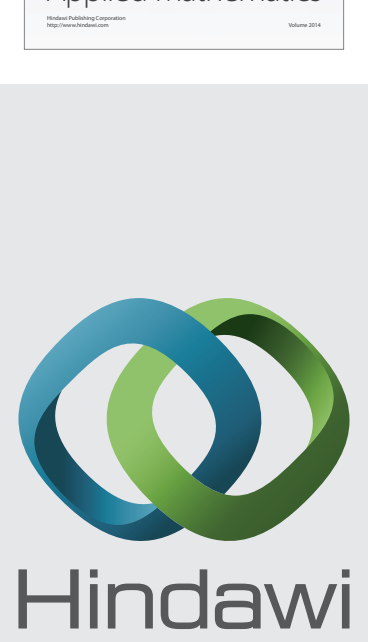

Submit your manuscripts at http://www.hindawi.com
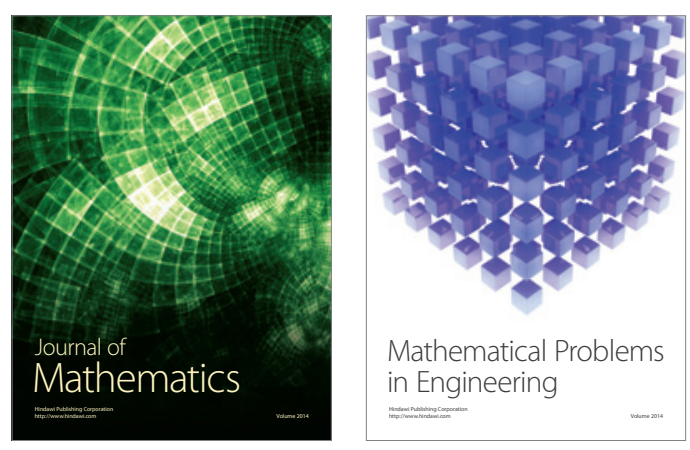

Mathematical Problems in Engineering
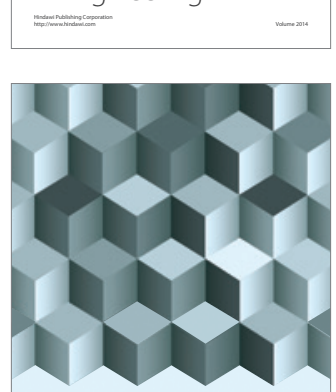

Journal of

Function Spaces
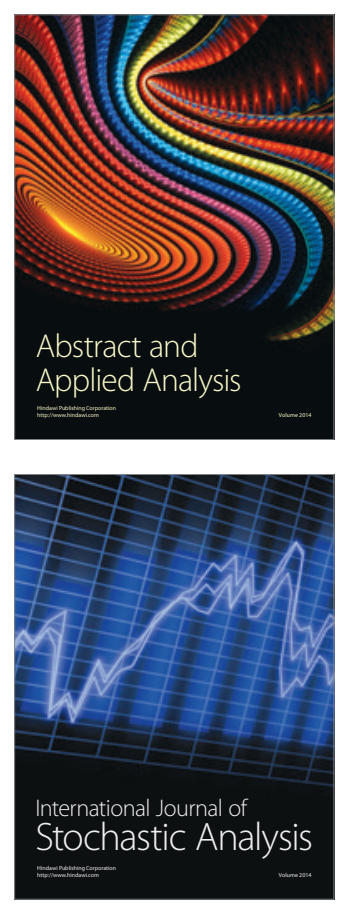

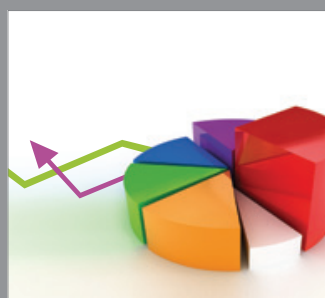

ournal of

Probability and Statistics

Promensencen
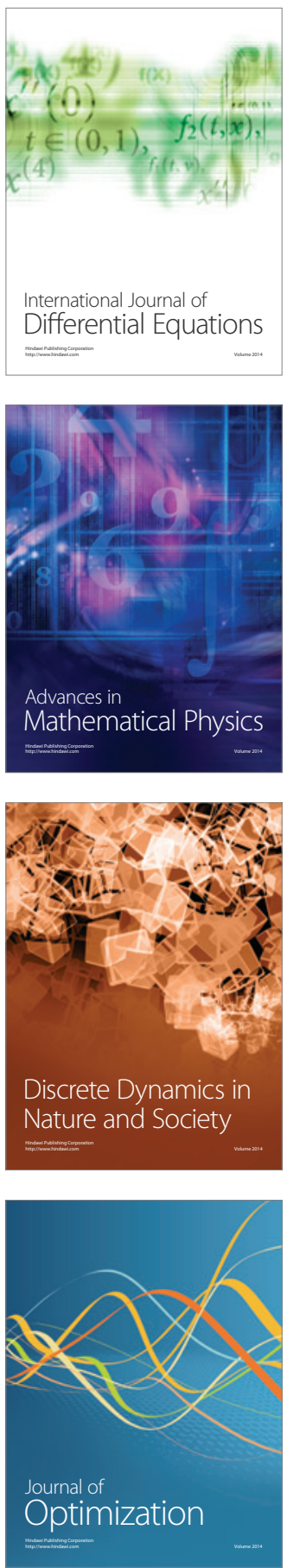